\title{
Factors affecting interprofessional teamwork in emergency department care of polytrauma patients: Results of an exploratory study
}

\section{Corresponding author:}

\author{
Alexandra LAPIERRE \\ RN, M. Sc., PhD student Université de Montréal, Montreal, Canada. \\ 1155 Henri-M-Perrault, Montréal, Québec, H1A 5K3 \\ Phone number: 1-514-963-0877 \\ Email address: alexandra.lapierre@umontreal.ca
}

\section{Co-authors:}

\section{Hélène, LEFEBVRE}

$\mathrm{RN}, \mathrm{PhD}$, University of Montreal, Montreal, Canada.

Faculty of Nursing, Université de Montréal, Pavillon Marguerite-d'Youville,

PO Box 6128, Downtown Station, Montreal, Quebec, Canada, H3C 3J7

Phone number: 1-514 343-6111 \#3382

Email address: helene.lefebvre@umontreal.ca

\section{Jérôme, GAUVIN-LEPAGE}

RN, Ph. D., University of Montreal, Montreal, Canada.

Faculty of Nursing, Université de Montréal, Pavillon Marguerite-d'Youville, PO Box 6128, Downtown Station, Montreal, Quebec, Canada, H3C 3J7

Phone number: 1-514-343-6111, ext. 26340, Fax number: 1-514-343-2306

Email address: jerome.gauvin-lepage@umontreal.ca

\section{Declaration of interest}

The authors report no declarations of interest. The authors alone are responsible for the content and writing of the paper.

This is the final version of the manuscript accepted for publication in Journal of Trauma Nursing. The published version is accessible here: https://doi.org/10.1097/jtn.0000000000000469 


\title{
Title:
}

Factors affecting interprofessional teamwork in emergency department care of polytrauma patients: Results of an exploratory study

\begin{abstract}
:
Considering that traumatic injuries are the leading cause of death among young adults across the globe, emergency department care of polytrauma patients is a crucial aspect of optimized care and premature death prevention. Unfortunately, many studies have highlighted important gaps in collaboration among different trauma team professionals, posing a major quality of care challenge. Using the conceptual framework for interprofessional teamwork (IPT) of Reeves, Lewin, Espin, and Zwarenstein (2010), the aim of this qualitative descriptive exploratory study was to better understand IPT from the perspective of health professionals in emergency department care of polytrauma patients, specifically by identifying factors that facilitate and impede IPT. Data was collected from a sample of seven health professionals involved in the care of polytrauma patients, through individual interviews and a focus group. In a second phase, two structured observations of polytrauma patient care were conducted. Following a thematic analysis, results show multiple factors affecting IPT, which can be divided into five broad categories: individual, relational, processual, organizational and contextual. Individual factors, a category that is not part of the conceptual framework of Reeves et al. (2010), also emerged as playing a major part in IPT.
\end{abstract}

\section{Keywords:}

Teamwork, polytrauma patient, traumatology, emergency care, qualitative study 


\section{Introduction}

Across the world, trauma-related injuries such as motor vehicle accidents, accidental falls or intentional injuries (i.e., self-injury and assault) are the leading cause of death among young adults and thus pose a serious global health problem (Haagsma et al., 2015). These types of injuries may cause widespread damage in patients and are clinically defined as polytraumatic when two or more traumatic lesions with respiratory or circulatory effects are present (Barsotti, 2010).

Because these polytraumatic injuries are so often life-threatening, initial care of polytrauma patients in emergency departments essentially aims to stabilize vital functions and prevent premature death (American College of Surgeons, 2018). Thus, the nature and speed of interventions could quite literally mean the difference between life and death (American College of Surgeons, 2018; Leonard, Graham, \& Bonacum, 2004). Studies have shown that interprofessional teamwork (IPT) plays an absolutely essential role in emergency trauma care settings as there seems to be a positive correlation between teamwork and clinical performance in trauma care teams (Briggs et al., 2015; Pucher, Aggarwal, Batrick, Jenkins, \& Darzi, 2014). Moreover, significant gaps in team communication and coordination in critical trauma care are among the main causes of preventable medical errors (Flin, Winter, Sarac, \& Raduma, 2009), such as avoidable delays in investigation and treatment procedures, and thus result in higher rates of complications and mortality (Chua, D'Amours, Sugrue, Caldwell, \& Brown, 2009; Lubbert, Kaasschieter, Hoorntje, \& Leenen, 2009).

In light of these findings, research on improving teamwork in the trauma setting has become more popular in recent years, mostly taking the form of intervention evaluations, team training and clinical simulations (Ajeigbe, McNeese Smith, Leach, \& Phillips, 2013; Gjerra, 2014). Yet, there is a growing trend in interprofessional healthcare teams as well as a new approach for improving treatment that is now based on understanding how health professionals can best work within teams as opposed to focusing on individual performance and behavior (Al Sayah, Szafran, Robertson, Bell, \& Williams, 2014). It follows that identifying factors that influence the quality of healthcare services provided by IPT may lead to significant impacts on both health professionals and their patients (Jacobson, 2012).

In the specific case of emergency room settings, very few studies have investigated how interprofessional teams provide polytrauma care and the factors that affect this process (Courtenay, Nancarrow, \& Dawson, 2013). 
In addition, many types of conceptual frameworks have been proposed to improve IPT in health care, but not all are created equal (for a review, see Tomizawa, Shigeta, and Reeves (2017). Reeves, Lewin, Espin, and Zwarenstein (2010) emphasize the importance of choosing a framework that addresses more than one dimension of interprofessional teamwork. Based on social sciences (i.e., social, psychological and organizational theories), this framework attempts to understand the nature and practices of IPT through empirical data. It focuses on improved knowledge of factors important to IPT, organized into four categories: relational, processual, organizational and contextual. This makes it ideally suited to our purpose.

\section{Study purpose}

The aim of this study was to identify the factors that facilitate and impede IPT according to health professionals working in emergency care of polytrauma patients in a secondary trauma center using the Reeves et al. (2010) conceptual framework as a theoretical and methodological reference model.

\section{Method}

\section{Design, setting and sample}

This study used a qualitative descriptive exploratory design, and was conducted at a hospital in Montreal, Canada, a designated secondary trauma center with approximately 1,000 trauma cases per year. The emergency department of this hospital was selected not only because of its access to polytrauma patients but also because of the interdisciplinary collaboration systems it offers. It should be noted that this hospital is not an academic health center and that, consequently, there are no medical students working within its different care units.

Participants were recruited through an invitation letter provided to all healthcare professionals working in the emergency department. Inclusion criteria for this study were as follows: 1) to be a healthcare professional; 2) have more than one year of experience in trauma care; and 3) have experienced more than one initial management of a polytraumatized patient at the emergency department. In addition, an explanatory letter was sent to all emergency personnel (via e-mail and memo) to explain the study and to notify of the possibility that the investigator could observe, at any time, interprofessional care of a trauma patient within the trauma room. Then, the investigator met the potential participants in person, taking the time to explain the purpose of the study, and their participation expectations. If they were interested in participating, the 
investigator made sure to contact them to schedule the appointment for the first individual interview.

The convenience sample consisted of seven participants $(n=7)$ representative of the interprofessional team usually involved with this clientele. These included a nurse, a respiratory therapist, an emergency physician, a head nurse assistant, an emergency nurse advisor, a paramedic and an orderly. These health professionals were chosen because of their involvement in the initial management of all polytraumatized patients in this emergency department (Table 1).

Table 1. Sociodemographic data and other relevant data obtained from participants

\begin{tabular}{|c|c|c|}
\hline Characteristics & \multicolumn{2}{|c|}{ Sample size $(n=7)$} \\
\hline Gender & Male: 2 & Female: 5 \\
\hline Age & $\begin{array}{l}20-29: 1 \\
30-39: 3\end{array}$ & $\begin{array}{l}40-49: 2 \\
50-59: 1\end{array}$ \\
\hline Education & DEP: 1 & $\begin{array}{l}\text { Collegial: } 3 \\
\text { University: } 3\end{array}$ \\
\hline Professional field & $\begin{array}{l}\text { Nurse: } 3 \\
\text { Emergency p } \\
\text { Respiratory tl } \\
\text { Orderly: } 1 \\
\text { Paramedic: } 1\end{array}$ & \\
\hline Number of years in practice & $\begin{array}{l}\text { 1-5 years: } 1 \\
\text { 6-10 years: } 2\end{array}$ & $\begin{array}{l}11-15 \text { years: } 1 \\
16+\text { years: } 3\end{array}$ \\
\hline $\begin{array}{l}\text { Number of years of experience in } \\
\text { trauma care }\end{array}$ & $\begin{array}{l}1-5 \text { years: } 2 \\
6-10 \text { years: } 1\end{array}$ & $\begin{array}{l}\text { 11-15 years: } 3 \\
16+\text { years: } 1\end{array}$ \\
\hline Relevant continuing education & ATLS: 3 & ACLS: 3 \\
\hline Current occupation & Full time: 6 & Part time: 1 \\
\hline Work shift & $\begin{array}{l}\text { Day: } 4 \\
\text { Evening: } 1\end{array}$ & $\begin{array}{l}\text { Night: } 1 \\
\text { Varies: } 1\end{array}$ \\
\hline
\end{tabular}




\section{Data collection}

Data was collected in three phases between June and November 2017 (Table 2). In the first phase, semi-structured individual interviews were conducted with each participant. An open question interview guide based on Reeves et al.'s (2010) framework was developed for these interviews. The guide focuses on the relational, processual, organizational, and contextual factors affecting IPT. Semi-structured interviews lasting approximately 30 to 45 minutes, recorded on digital audio and transcribed verbatim, were conducted outside working hours. In addition, a sociodemographic data survey was completed by all participants.

In the second phase, two direct observations of polytrauma patient care were then carried out using an observation checklist previously developed with the assistance of two experts. Notably, this checklist allows classification of factors into the four categories in the Reeves et al. (2010) conceptual framework. Observations were recorded in descriptive and neutral terms directly on this checklist. Because it is impossible to predict patient arrival beyond a few minutes, the subjects of observation of polytrauma patient care were the health professionals present at that time.

Finally, in phase three, after initial data analysis, the seven study participants were invited to take part in a focus group. A second interview guide was also developed for this last phase. In the focus group, a summary of results obtained in the first analysis was presented to participants as a slideshow. During this presentation, participants were asked to complete, confirm or refute statements made in individual interviews. The focus group, which lasted 90 minutes, was also recorded on digital audio and then transcribed verbatim for analysis.

Table 2. Data collection summary

\begin{tabular}{|l|l|l|}
\hline Date collection phase & \multicolumn{1}{|c|}{ Data collection method } & \multicolumn{1}{c|}{ Total time } \\
\hline Phase 1 & 7 semi-structured interviews & 285 minutes (4.75 hours) \\
\hline Phase 2 & 2 observation periods & 80 minutes (1.3 hours) \\
\hline Phase 3 & Focus group & 90 minutes (1.5 hours) \\
\hline
\end{tabular}

\section{Data analysis}

The data analysis process was based on Miles and Huberman (2003). These authors propose three steps: data reduction, data display, and conclusion drawing and verification. 
In step 1 (data reduction), all data from the semi-structured individual interviews and the structured direct observations conducted during polytrauma patient care were consolidated. For every individual interview and observation note, initial coding was performed by the main investigator of the study using several approaches, namely descriptive, interpretative and explanatory. Data was then grouped or divided thematically using the MAXQDA analysis program (version 12.0). Finally, co-investigators of the study read and validated the coded data.

In step 2 (data display), data was organized so it made sense when presented. As a reminder, the third phase of the study consisted of a focus group wherein an initial summary of data analysis results was presented to participants in table format in order to confirm or refute collected data. Following feedback from the focus group, a second analysis was performed to triangulate data related to the individual interviews, the observation data, and the focus group.

In the third and final step (drawing and verification of conclusions), verification of conclusions was conducted with study participants in the focus group and with co-investigators, who read the analyses and validated and confirmed results.

\section{Ethical considerations}

The research protocol was submitted to the ethics committee of the hospital where the study was to take place and subsequently approved (study \#2018-1039). Thus, this study complies with the standards set out in the Tri-Council Policy Statement regarding free and informed consent, privacy and confidentiality, conflicts of interest, and protection from potential harm of study participants (Canadian Institutes of Health Research, Natural Sciences and Engineering Research Council of Canada, \& Social Sciences and Humanities Research Council of Canada, 2014).

\section{Results}

Factors that facilitate and impede IPT in the trauma setting were divided according to the four categories described in Reeves et al.'s conceptual framework (2010), i.e., relational, processual, contextual, and organizational. Interestingly, our data analysis yielded a fifth category of factors affecting IPT, namely individual factors. Figure 1 summarizes the results of this study. 
Figure 1. Factors that facilitate and impede IPT in the trauma setting

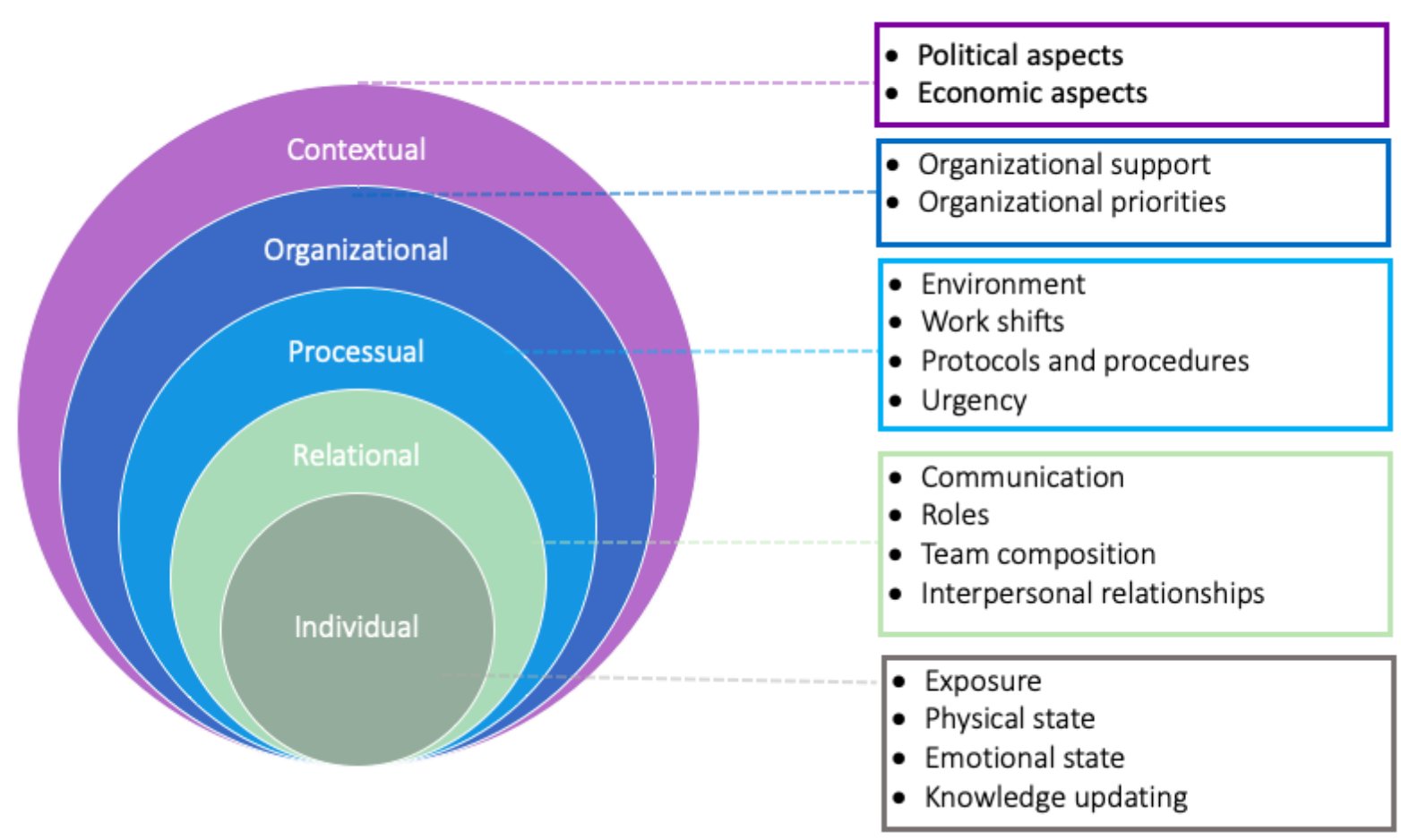

\section{Individual factors}

Exposure to polytrauma care. Among the interviewed health professionals, past frequent exposure to emergency polytrauma care is a facilitating factor. "I feel that people with prior exposure to polytrauma patient care are calmer and more comfortable in their role, and that communication is really more effective," stated the nurse advisor (focus group). Conversely, low exposure to emergency polytrauma patient care is a factor that impedes IPT. As several participants emphasized: "Also, the lack of experience of nurses. (...) They have trauma room training, of course, but when you haven't experienced it yourself, it's a little harder" (interview, head nurse assistant).

Physical and emotional state. According to health professionals, fatigue is a factor that impedes IPT. One participant stated, "When you're tired, you're less patient and you become stressed more easily, so it's hard" (interview, emergency physician). Moreover, interviewed professionals consider that the emotional state, i.e., the individual's mood and stress level, positively or negatively influences IPT. "Personal mood is also a factor. Let's say I'm here one 
morning and I'm feeling off, maybe I won't feel like collaborating (...)" (interview, respiratory therapist). With regard to stress, another participant mentioned: "Of course, some people are a little more stressed (...) in these types of situations, so they lose a little more control" (focus group, orderly), related to how they interact with trauma team members.

Knowledge updating. For trauma professionals, knowledge updating is a factor that seems to affect teamwork: "We have a responsibility to update our knowledge (...) on an individual level, but if people don't update their knowledge and stop training, it becomes a problem (...)" (interview, nurse advisor). In fact, it seems that keeping knowledge up-to-date influences the way health care is provided when treating a polytraumatized person. Even though the nurse advisor was the only one to identify this element in her interview, all health professionals in the focus group confirmed the relevancy of this factor.

\section{Relational factors}

Communication. Communication is a key component of teamwork for all professionals interviewed. Active and real-time communication in polytrauma care is a sub-theme identified by these professionals when referring to "good communication." Moreover, closed-loop communication appears to facilitate teamwork. The primary aim of this communication technique is to avoid misunderstandings by continually ensuring that what is said is properly understood and carried out. "I like it when instructions are repeated to make sure we understand what we are doing, or what the doctor wants (...), "stated the nurse advisor during the focus group. Inversely, chaotic communication, which may occur in emergencies involving polytraumatic injuries, was reported as an impeding factor. "It can get loud (...). The more noise there is, the louder doctors need to speak, the more chaotic it becomes," said the nurse (interview). In addition, the respiratory therapist explains in interview that: "When everyone is talking at the same time, it is difficult sometimes to hear the instructions (...). It can become cacophonous." According to participants, debriefing, i.e., when health professionals meet to review polytrauma care, is another strategy that positively affects IPT.

Roles. Role distribution seems to be another important factor in trauma care. It involves identifying each person's role, when possible, prior to the polytrauma patient's arrival. According to various health professionals, knowing one's role and that of other team members appears to be a facilitating factor. The paramedic stated: "You have to know your role, (...) the respiratory 
therapist's role, and the nurse's role" (interview) while the nurse advisor makes the following observation about polytrauma patient care: "This past year, I observed a lack of understanding of each other's roles" (interview). Sometimes, despite good distribution and understanding of roles, failing to maintain one's role in polytrauma patient care can impede IPT. Finally, identifying a leader seems to be an essential step in the trauma setting, both on the medical and nursing level. As noted by the assistant head nurse: "It is important to have a designated leader, to know who to listen to" (interview). With regards to her own role, she added: "When I'm an assistant, I'm the lead nurse, and among doctors, there's a lead doctor. We communicate with each other" (interview).

Team composition. As previously mentioned, having a single leader in polytrauma patient care is a facilitating factor according to professionals. "It takes one leader," the emergency physician himself stated in the focus group. However, during phase two of the study, observations revealed that there were in fact several medical leaders for each patient. These observations seem to be in line with reports of the participating physician, who agrees there is room to improve teamwork between physicians: "It is perhaps between doctors that it is not always clear" (interview, emergency physician).

Another factor appearing to have a positive effect on teamwork is the stability of the team. Health professionals define a stable team as one composed of people who know each other and are used to working together. Yet, in all departments, and especially in nursing, new health professionals are integrated into the different teams several times a year. As a result, teams are often made up of "new nurses," i.e., nurses with low trauma room experience. According to participants, integrating new staff into the trauma room affects teamwork, but seems to have no impact a when other experienced nurses are on site to support new recruits.

Interpersonal relationships. Not surprisingly, good interpersonal skills were reported by participants to be a facilitating factor. Effects of interpersonal conflicts, however, are ambiguous. One participant said: "Better interpersonal relationships usually result in better interprofessional relationships" (interview, physician). Participants stated that respect, mutual help, and trust are three components of positive interpersonal relationships: "I think team members who know and trust each other will be more "relaxed" even in a critical polytrauma case" (interview, paramedic). However, conflicts between professionals seem to have little or no impact on teamwork in polytrauma care. Indeed, this idea was raised by all and illustrated in the following quote: 
"If I have a conflict with someone, and I have a patient in cardiorespiratory arrest in front of me, and I'm working with this person, well then I'm working as a team with them. These kinds of conflicts disappear (...), you have a role to play. Here, you don't question it" (interview, nurse).

\section{Processual factors}

Environment. Physical environment and equipment also seem to have an impact on teamwork in polytrauma care. For example, when equipment is obsolete, missing, or even sometimes difficult to access, it appears to impede IPT. As one participant confessed: "We get frustrated, there is a lot of unfamiliar or misplaced equipment" (focus group, nurse). Moreover, the presence of non-trauma patients in the trauma room environment is a factor that impacts teamwork in polytrauma care. According to participants, family presence in the room affects teamwork. Indeed, even though participants believe that families have a right to be in the room, they also think family members must be accompanied by a health worker so as not to negatively impact events. "Family presence doesn't bother me, if they are calm, and are accompanied by hospital staff who can supervise them," said the physician (focus group). However, data collected from observation of two polytrauma patients revealed that no family members were welcome during patient emergency care.

Work shifts. According to participants, trauma practice varies depending on the shift. Indeed, it seems that there are significant differences between the three shifts: "It's a different reality during the day, evening and night, as resources are not the same and the experience is not the same either" (interview, nurse advisor). Furthermore, a focus group participant stated that: "The day shift is easier, because you have the old gang that you can pair with new beginner staff. This is not a luxury you're afforded during the evening shift, although there are still a few senior staff members. On the night shift, forget it" (interview, nurse).

Protocols and procedures. Knowing trauma protocols and procedures seems to be a factor that positively impacts teamwork: "Procedures are important, especially for the leader. For example, knowing how to get packed red blood cells, how to coordinate with radiology, ATLS roles, it's really very important" (interview, assistant head nurse). Yet it seems protocols and procedures are sometimes not known and sometimes not respected, which naturally has a negative impact on teamwork. For example, the respiratory therapist stated that he was not informed of the 
arrival of polytrauma patients on several occasions, which is a required procedure. Moreover, interhospital transfers sometimes seem problematic, in the sense that collaboration between hospitals is complicated and inefficient. In point of fact, the orderly described a transfer that went rather badly: “The other center wasn't collaborating. There were lots of delays, and it could have gone a lot faster" (interview).

Also, at the processual level, deficient technical support coordination can negatively impact teamwork in the trauma setting, according to several health professionals. The assistant head nurse explained that technical support coordination is not always successful, and that it often causes delays.

Lastly, preparation seems to be a facilitating factor of this category, making it essential that pre-hospital staff give notice of patient arrival. In addition to advance notification of incoming patients, trauma room preparation is also important, according to study participants.

Urgency. Severity of patient injuries appears to be a factor that impacts teamwork, in that the more critical or unstable the patient is, the more difficult teamwork becomes. The paramedic reported: "The more acute the patient's condition, the higher the stress levels" (interview). For the nurse advisor, age also seems to have an impact on the sense of urgency: "Young patients cause more stress, and I don't mean to say a 90-year-old isn't important, but I believe that we're more nervous (...) when the trauma patient is in their twenties" (focus group).

\section{Organizational factors}

Organizational support. Human and material resources provided by the organization necessarily seem to impact teamwork. Also, the nurse describes the sense of belonging that naturally facilitates the work of health professionals: "I think that when you're reassured and supported by your establishment, and that you feel good and grounded where you are, it's easier to work" (interview). Moreover, the clinical support provided to professionals in their clinical practice is a facilitating factor, as noted by the same participant: "We get a lot of support (...) from clinicians, supervisors and doctors when new things spring up along the way" (interview). Conversely, lack of clinical support during evening and night shifts seems to have a negative impact. In addition, participants mentioned that training sessions also impact teamwork, by making it possible to improve as a team in a context outside of real practice. 
Organizational priorities. Participants discussed their impressions about the ranking of their trauma unit in their organization's priorities. "If trauma isn't a priority, I think it can impede skill development for nurses, doctors, staff, respiratory therapists, technical services, everyone involved," emphasized the nurse advisor in interview. Another concern reported by several health professionals is feeling that the organization is very focused on performance markers, i.e., statistics. They can't say whether this is true, but they claim to often have had the following impression: "We're very much about performance markers (...). If they've been reached, it would seem that the "rest" matters less!" (focus group, paramedic).

\section{Contextual factors}

Political aspects. One participant stated: “(...) Ministry of Health administrative changes create a lot of uncertainty" (interview, physician). At the political level, the relationship between different partners is not always facilitating. Also, the relationship between different services does not seem optimal. As stated by the paramedic: "I think the relationship between partners is weak. You're not informed of our changes, and when we get there, you don't understand what we're doing" (regarding the relationship between paramedical care and trauma hospital organization) (interview).

Economic aspects. Many professionals mention the impact of both human and material cuts. "We're always tightly staffed. If they can take one out, they take one out," said the respiratory therapist, referring to respiratory therapists (interview). Meanwhile, the doctor observed a lack of physicians in his department: "We don't have enough doctors, and it causes a lot of problems because everyone works more than $100 \%$ of their maximum, so it's difficult" (interview). Health professionals are also affected by material cuts, which indirectly impact trauma team collaboration: "For example, we had a nice fluid warmer, but we don't have it anymore. It's worth $\$ 10,000$, and they don't want to replace it" (focus group, nurse advisor).

\section{Discussion}

The results of this study seem to support previous findings in the literature on factors affecting IPT in the trauma setting, notably on individual, relational and processual factors, which will be discussed below. Of particular note, however, are the new results on organizational and contextual factors affecting teamwork in the trauma setting yielded by this study. 
In terms of individual factors, our data supports the existing literature on the impact of professionals' trauma experience (Cole \& Crichton, 2006; Khademian et al., 2013) and individual characteristics such as stress, fatigue and distractions on teamwork and performance (Barach \& Weinger, 2007; Sexton, Thomas, \& Helmreich, 2000; Xiao, Parker, \& Manser, 2013). As for knowledge updating, several papers note that professionals' ability to collaborate successfully and be part of a team is necessarily affected by individual knowledge, attitude and skills that may all be improved through training (Khademian et al., 2013; Leggat, 2007).

A central finding of this study concerns the important influence interactions between team members (i.e., relational factors) have on teamwork. Indeed, this was the most salient theme brought up by participants. This finding is also in line with several past studies reporting that good communication (i.e., clear and precise communication in which listening is emphasized), is fundamental to the success of a healthcare team (Cole \& Crichton, 2006; Raley et al., 2016; Westli, Johnsen, Eid, Rasten, \& Bratteb $\varnothing, 2010$ ), which also includes transfer information from prehospital staff upon arrival at the ER (Wood, Crouch, Rowland, \& Pope, 2015). In fact, the use of communication techniques, such as closed-loop communication, in the trauma setting has been deemed crucial in preventing errors (El-Shafy et al., 2018; Härgestam, Lindkvist, Brulin, Jacobsson, \& Hultin, 2013; Jung et al., 2018). The debriefing period also seems essential according to participants and Steinemann et al. (2016), since it is a good time for knowledge sharing and team building.

Our research also highlights the importance of leadership in the trauma setting, since the participants believe that the presence of a medical leader and a nursing leader is essential for group cohesion. Here also, several authors confirm that the presence of an effective leader is correlated with increased team collaboration in both the medical and nursing context (Clements, Curtis, Horvat, \& Shaban, 2015; Ford et al., 2016). Indeed, participants report that teamwork between physicians is often difficult, in the sense that all physicians on the floor simultaneously take on the role of leader, resulting in confusion for other team members. Sarcevic, Marsic, Waterhouse, Stockwell, and Burd (2011) have shown that this type of leadership structure, where two physicians act independently from each other, is in fact the most harmful for the team. The concept of stability of team members raised by participants in this study is also consistent with past research reporting that a stable or shifting team composition affects teamwork (Kendall-Gallagher, Reeves, Alexanian, \& Kitto, 2017; Xiao et al., 2013). 
Notably, participants in this study raised a new issue, to our knowledge, concerning team composition. To wit, the integration of new professionals as a factor that affects teamwork. For participants, "new" refers to nurses with no trauma room experience as opposed to novice nurses. Nevertheless, this seems like a plausible finding considering the literature on new graduate nurses, which fully supports participants' comments on the positive impact of professional coaching and support for new nurse integration (Pfaff, Baxter, Jack, \& Ploeg, 2014).

Finally, our findings on the impact of respect, trust and mutual support in interpersonal relationships on team dynamics and job satisfaction is also in line with past literature (KendallGallagher et al., 2017; Khademian et al., 2013; Lee \& Doran, 2017). However, the effects of interpersonal conflicts in the trauma setting do not seem to have been addressed in the literature. This seems like an important avenue for further research, as in other care settings, it appears that interpersonal conflicts strongly impact teamwork, with several negative consequences, primarily on patient care (Bochatay et al., 2017; Patton, 2014). The results of this study therefore diverge from the literature, in that interviewed professionals believe that interpersonal conflicts have no impact on teamwork in emergency situations such as polytrauma care. These results might differ from those observed in other care settings because of the life and death urgency of emergency polytrauma care units. As participants noted, personal grievances seem trivial when one is trying to save a life. One caveat, however, is that participants did not address the impact of interpersonal conflicts on their daily clinical practice, which, as previously stated, is probably much different than their emergency work with polytraumatic patients.

The processual factors that affected IPT in the trauma setting according to our data were the environment (in terms of presence or absence of people and equipment), work shifts, protocols and procedures, as well as urgency. Participants in our study echo previous research emphasizing the fact that an adequate physical environment and access to suitable equipment have an impact on teamwork in emergency rooms (Gharaveis, Hamilton, \& Pati, 2017; Khademian et al., 2013). Noise and number of people (e.g., professionals and patients) present in the room are other factors affecting teamwork and effective communication (Cole \& Crichton, 2006; Wood et al., 2015). Similarly, presence and number of family members is another factor that must be taken into account according to our data. However, the true impact of this factor remains rather unclear, as, in the course of phase two observations, family was not invited to remain at the patient's bedside. The literature does seem to address this contradiction. In fact, two currents of thought seem prevalent 
among health professionals. Some believe that the presence of family members reduces effectiveness of care by creating distractions, whereas others maintain that family at patients' side is beneficial, as they can see what is being done to save their loved one's life (Twibell, Siela, Riwitis, Neal, \& Waters, 2018). Thus, the discrepancy in results may be due to the fact that professionals observed in phase 2 did not hold the same views as those who participated in the individual interviews and the focus group.

Health professionals in this study also highlighted work shifts as a factor affecting teamwork in trauma practice. Indeed, resources and personnel experience differ according to the shift, with night shifts being staffed with more inexperienced personnel and day shifts having the best ratio of experienced to inexperienced staff.

Not surprisingly, a structured work environment, where protocols and procedures are adhered to and respected, helps facilitate communication and teamwork, according to both our sample and previous research (Weller, Boyd, \& Cumin, 2014; Wood et al., 2015). Not following protocols and procedures has the opposite effect, by increasing health professionals' stress levels, which in turn impacts the efficacy of teamwork (Khademian et al., 2013).

Again, in line with previous research findings, participants reported preparation of the physical space and of the team members before a patient is admitted as being a facilitating factor in trauma practice (Khademian et al., 2013).

Inter-hospital transfers also impact care team performance, as patient transfer to a center specialized in trauma care requires impeccable coordination and communication between all involved in order to provide safe, quality care (Rokos, Sanddal, Pancioli, Wolff, \& Gaieski, 2010; Sethi \& Subramanian, 2014). Participants also underscored the importance of internal coordination of different technical services as a rather constraining factor, in that processes are not always efficient due to the simultaneous involvement of multiple services and constant changes in care procedures (Fisher, Weyant, Sterrett, Ambrose, \& Apfel, 2017).

Furthermore, a sense of urgency, specifically as it pertains to patient condition, seems to have a strong impact on team cooperation and coordination. This was true both in our study and in Cole and Crichton (2006) paper, in which it appeared that the more a patient is unstable, the more teamwork is described as chaotic. Participants in our study went further still in their observations, reporting that the patient's age also has an impact on their sense of urgency, which has only been documented in the context of cardiovascular resuscitation (Frost, Cook, Heyland, \& Fowler, 2011). 
Whereas the added sense of pressure according to the patient's age reported in the trauma care unit seems to be about saving the patient's life, in the case of cardiovascular resuscitation, the sense of urgency is tied to decision-making in ending resuscitation maneuvers (Frost et al., 2011).

Organizational factors identified in this study mainly had to do with institutional support and priorities. These factors encompass several elements, namely human and material resources, clinical support, training, as well as a sense of belonging. This is consistent with recent findings in trauma and critical care research, which also emphasize the importance of human and material resources for quality of care provided (Kendall-Gallagher et al., 2017; Khademian et al., 2013). Moreover, according to several critical care researchers, clinical support and interprofessional education are two other factors that promote teamwork (Fisher et al., 2017; Goldman, Kitto, \& Reeves, 2017; Weller et al., 2014). Availability of resources is tied to organizational priorities, which thus becomes another factor considered important by our sample. In fact, participants' perceptions that teamwork and trauma are not priorities negatively impact teams in this clinical setting. San Martín-Rodríguez, Beaulieu, D'Amour, and Ferrada-Videla (2005) also point out that all care settings require a functioning organizational framework, with a supportive structure and culture, to promote teamwork. Moreover, participants also shared their impressions that the organization is strongly committed to performance markers. This may be due to the fact that the organization must gauge trauma procedures and results. Indeed, there are many studies on team performance assessment, as it is impossible to gauge performance improvement without it (Georgiou \& Lockey, 2010; Lubbert et al., 2009). Be that as it may, for interviewed health professionals, this perception appears to have a negative impact on teamwork.

Political and economic aspects are predominant contextual factors, according to study participants. Since 2014, health system reform in Quebec (Canada) seems to have had an impact on healthcare practice. In the hospital center in which these professionals work, it resulted in a merger with several other institutions in the territory. Indeed, these health professionals believe that the changes to the current health system, which resulted in significant budgetary restrictions, impact teamwork in trauma care because cuts have a concrete effect on both the human and material level.

At the political level, participants denounce shortcomings in relationships between various instances involved in the care of polytraumatic patients, especially as it pertains to pre-hospital and hospital personnel. If these types of factors are not addressed in the trauma care literature, research 
in similar care settings, such as intensive care (Kendall-Gallagher et al., 2017) and primary care (O’Reilly et al., 2017), has identified economic and political aspects as factors affecting teamwork. O'Reilly et al. (2017) highlight that financial resources are extremely significant for teamwork because they determine team composition, training opportunities, communication systems, and even the physical space available.

\section{Implications for trauma nurses}

Nurses are an integral part of all healthcare settings. Results obtained in this study are specific to trauma care nurses, yet are consistent with results of research in other care settings

investigating factors affecting interprofessional teamwork (see Al Sayah et al. (2014)). This study may further serve to emphasize the importance of teamwork, as well as the key role that nurses play in its effectiveness (for example, at the individual, relational, processual, and even organizational level). Indeed, in light of these findings, nurses may want to invite their team members to reflect on facilitating and impeding factors influencing teamwork and perhaps consider implementing some of the participant's recommendations (i.e. facilitating factors) reported in this study. Given that nurses are an important part of interdisciplinary teams, effective preparation of these professionals on efficient teamwork practices may benefit overall quality of care for trauma patients.

\section{Strengths and limitations}

The study's first strength lies in the choice of the conceptual framework, on which the methodology for data collection, analysis, and interpretation of results were based. Its second strength is the multidisciplinarity and heterogeneity of our sample. In fact, input from a paramedic, a respiratory therapist, and an orderly is, to our knowledge, unique yet yields important insights into interdisciplinary teamwork. With this strength, however, comes an inherent limitation, namely sample size $(n=7)$, which makes results difficult to generalize to all professionals represented by a single individual. Nevertheless, the purpose of this study was not to compare, and contrast perspectives of different professionals involved in IPT in order to generate statistical analyses, but rather to obtain a dataset from a trauma care team. Moreover, despite sample size, many of the findings reported here are corroborated extensively by the scientific literature of the past decade. The study's third strength lies in our methodology, namely the use and triangulation of multiple 
data collection methods: the individual interview, the focus group, and direct observation. This may help to mitigate a final limitation, which comes from the fact that the main investigator of this study is herself a nurse in the emergency trauma unit, which could have had an impact on her interpretation of results.

\section{Conclusion}

Through the diversity of professionals represented in its sample, this study yields insight into the actual reality of IPT in trauma care units. Factors brought up by participants here echo past research findings on the subject of teamwork in healthcare settings, namely regarding individual, relational and processual factors. Results confirm that there are multiple factors impacting all levels of collaboration in trauma care and affecting health professionals from all angles. Importantly, this study also generated new organizational and contextual factors affecting teamwork in the trauma setting. Indeed, this trauma study is the first in the literature to address factors of teamwork related to contextual aspects.

In short, these results will possibly lead to a better understanding of the realities of emergency department care of polytrauma patients as experienced by actual interprofessional teams. In a time where multidisciplinary teams are increasingly becoming the norm in healthcare settings, research into the reality and particular needs of professionals in specific care units seems essential. It is hoped that this data will serve as a tool for further reflection by professionals on their collaboration practices in trauma units, in order to improve IPT. In this way, the contribution of this paper may lie in the development of specific interventions that aim to improve teamwork in the management of polytrauma patients at the emergency department. 


\section{References}

Ajeigbe, D., McNeese Smith, D., Leach, L., \& Phillips, L. (2013). Nurse-physician teamwork in the emergency department: impact on perceptions of job environment, autonomy, and control over practice. The Journal of nursing administration, 43(3), 142-148. doi:10.1097/NNA.0b013e318283dc23

Al Sayah, F., Szafran, O., Robertson, S., Bell, N. R., \& Williams, B. (2014). Nursing perspectives on factors influencing interdisciplinary teamwork in the Canadian primary care setting. Journal of Clinical Nursing, 23(19-20), 2968-2979. doi:10.1111/jocn.12547

American College of Surgeons. (2018). ATLS Advanced Trauma Life Support : student course manual (10 ed.). Chicago, United-States: American College of Surgeons.

Barach, P., \& Weinger, M. B. (2007). Trauma team performance. In W. C. Wilson, G. C.M., \& D. B. Hoyt (Eds.), Trauma: Emergency Resuscitation, Perioperative Anesthesia, Surgical Management (Vol. 1, pp. 101-111). Florida: CRC Press.

Barsotti, J. (2010). Le polytraumatisé (approche pratique). In J. Cancel, C. Robert, V. Martinel, A. Cernier, É. Gaisne, \& P. Lermusiaux (Eds.), Guide pratique de traumatologie (6 ed., pp. 306-309). Paris: Elsevier Masson.

Bochatay, N., Bajwa, N. M., Cullati, S., Muller-Juge, V., Blondon, K. S., Junod Perron, N., . . . Nendaz, M. R. (2017). A multilevel analysis of professional conflicts in health care teams: insight for future training. Academic Medicine, 92(11S), S84-S92. doi:10.1097/acm.0000000000001912

Briggs, A., Raja, A. S., Joyce, M. F., Yule, S. J., Jiang, W., Lipsitz, S. R., \& Havens, J. M. (2015). The role of nontechnical skills in simulated trauma resuscitation. Journal of Surgical Education, 72(4), 732-739. doi:10.1016/j.jsurg.2015.01.020

Canadian Institutes of Health Research, Natural Sciences and Engineering Research Council of Canada, \& Social Sciences and Humanities Research Council of Canada. (2014). TriCouncil policy statement: ethical conduct for research involving humans. Ottawa, Canada: Gouvernement du Canada

Chua, W. C., D'Amours, S. K., Sugrue, M., Caldwell, E., \& Brown, K. (2009). Performance and consistency of care in admitted trauma patients: our next great opportunity in trauma care? ANZ Journal of Surgery, 79(6), 443-448. doi:10.1111/j.1445-2197.2009.04946.x

Clements, A., Curtis, K., Horvat, L., \& Shaban, R. Z. (2015). The effect of a nurse team leader on communication and leadership in major trauma resuscitations. International Emergency Nursing, 23(1), 3-7. doi:10.1016/j.ienj.2014.04.004

Cole, E., \& Crichton, N. (2006). The culture of a trauma team in relation to human factors. Journal of Clinical Nursing, 15(10), 1257-1266. doi:10.1111/j.1365-2702.2006.01566.x 
Courtenay, M., Nancarrow, S., \& Dawson, D. (2013). Interprofessional teamwork in the trauma setting: a scoping review. Human Resources for Health, 11(1), 1-10. doi:10.1186/14784491-11-57

El-Shafy, I. A., Delgado, J., Akerman, M., Bullaro, F., Christopherson, N. A. M., \& Prince, J. M. (2018). Closed-loop communication improves task completion in pediatric trauma resuscitation. Journal of Surgical Education, 75(1), 58-64. doi:10.1016/j.jsurg.2017.06.025

Fisher, M., Weyant, D., Sterrett, S., Ambrose, H., \& Apfel, A. (2017). Perceptions of interprofessional collaborative practice and patient/family satisfaction. Journal of Interprofessional Education \& Practice, 8, 95-102. doi:10.1016/j.xjep.2017.07.004

Flin, R., Winter, J., Sarac, C., \& Raduma, M. (2009). Human factors in patient safety: review of topics and tools. Switzerland: World Health Organisation

Ford, K., Menchine, M., Burner, E., Arora, S., Inaba, K., Demetriades, D., \& Yersin, B. (2016). Leadership and teamwork in trauma and resuscitation. The Western Journal of Emergency Medicine, 17(5), 549-556. doi:10.5811/westjem.2016.7.29812

Frost, D. W., Cook, D. J., Heyland, D. K., \& Fowler, R. A. (2011). Patient and healthcare professional factors influencing end-of-life decision-making during critical illness: A systematic review. Critical Care Medicine, 39(5), 1174-1189. doi:10.1097/CCM.0b013e31820eacf2

Georgiou, A., \& Lockey, D. J. (2010). The performance and assessment of hospital trauma teams. Scandinavian Journal of Trauma, Resuscitation and Emergency Medicine, 18(1), 66. doi:10.1186/1757-7241-18-66

Gharaveis, A., Hamilton, D. K., \& Pati, D. (2017). The impact of environmental design on teamwork and communication in healthcare facilities: a systematic literature review. HERD: Health Environments Research \& Design Journal, 11(1), 119-137. doi:10.1177/1937586717730333

Gjerra, K., Moller, T.P. et Ostergaard, D. . (2014). Efficacy of simulation-based trauma team training of non-technical skills. A systematic review. Acta Anaesthesiolica Scandinavica, 58, 775-787. doi:10.1111/aas.12336

Goldman, J., Kitto, S., \& Reeves, S. (2017). Examining the implementation of collaborative competencies in a critical care setting: Key challenges for enacting competency-based education. Journal of Interprofessional Care, 1-9. doi:10.1080/13561820.2017.1401987

Haagsma, J. A., Graetz, N., Bolliger, I., Naghavi, M., Higashi, H., Mullany, E. C., . . Vos, T. (2015). The global burden of injury: incidence, mortality, disability-adjusted life years and time trends from the Global Burden of Disease study 2013. Injury Prevention. doi:10.1136/injuryprev-2015-041616 
Härgestam, M., Lindkvist, M., Brulin, C., Jacobsson, M., \& Hultin, M. (2013). Communication in interdisciplinary teams: exploring closed-loop communication during in situ trauma team training. BMJ Open, 3(10), 1-8. doi:10.1136/bmjopen-2013-003525

Jacobson, P. M. (2012). Evidence synthesis for the effectiveness of interprofessional teams in primary care. Ottawa, ON: Canadian Health Services Research Foundation

Jung, H. S., Warner-Hillard, C., Thompson, R., Haines, K., Moungey, B., LeGare, A., . . Sullivan, S. (2018). Why saying what you mean matters: An analysis of trauma team communication. The American Journal of Surgery, 215(2), 250-254. doi:10.1016/j.amjsurg.2017.11.008

Kendall-Gallagher, D., Reeves, S., Alexanian, J. A., \& Kitto, S. (2017). A nursing perspective of interprofessional work in critical care: Findings from a secondary analysis. Journal of Critical Care, 38, 20-26. doi:10.1016/j.jcrc.2016.10.007

Khademian, Z., Sharif, F., Tabei, S., Bolandparvaz, S., Abbaszadeh, A., \& Abbasi, H. (2013). Teamwork improvement in emergency trauma departments. Iranian Journal of Nursing and Midwifery Research, 18(4), 333-339.

Lee, C. T.-S., \& Doran, D. M. (2017). The role of interpersonal relations in healthcare team communication and patient safety: a proposed model of interpersonal process in teamwork. Canadian Journal of Nursing Research, 49(2), 75-93. doi:10.1177/0844562117699349

Leggat, S. G. (2007). Effective healthcare teams require effective team members: defining teamwork competencies. BMC Health Services Research, 7, 17-17. doi:10.1186/14726963-7-17

Leonard, M., Graham, S., \& Bonacum, D. (2004). The human factor: the critical importance of effective teamwork and communication in providing safe care. Quality \& safety in health care, 13(Suppl 1), i85-i90. doi:10.1136/qshc.2004.010033

Lubbert, P. H., Kaasschieter, E. G., Hoorntje, L. E., \& Leenen, L. P. H. (2009). Video registration of trauma team performance in the emergency department: the results of a 2-year analysis in a level 1 trauma center. The Journal of Trauma, 67(6), 1412-1420. doi:10.1097/TA.0b013e31818d0e43

O’Reilly, P., Lee, S. H., O’Sullivan, M., Cullen, W., Kennedy, C., \& MacFarlane, A. (2017). Assessing the facilitators and barriers of interdisciplinary team working in primary care using normalisation process theory: An integrative review. PLOS ONE, 12(7), e0181893. doi:10.1371/journal.pone.0177026

Patton, C. M. (2014). Conflict in health care: a literature review. The Internet Journal of Healthcare Administration, 9(1), 1-11. Retrieved from https://print.ispub.com/api/0/ispubarticle/20081.

Pfaff, K., Baxter, P., Jack, S., \& Ploeg, J. (2014). An integrative review of the factors influencing new graduate nurse engagement in interprofessional collaboration. Journal of Advanced Nursing, 70(1), 4-20. doi:10.1111/jan.12195 
Pucher, P. H., Aggarwal, R., Batrick, N., Jenkins, M., \& Darzi, A. (2014). Nontechnical skills performance and care processes in the management of the acute trauma patient. Surgery, 155(5), 902-909. doi:10.1016/j.surg.2013.12.029

Raley, J., Meenakshi, R., Dent, D., Willis, R., Lawson, K., \& Duzinski, S. (2016). The role of communication during trauma activations: investigating the need for team and leader communication training. Journal of Surgical Education, 74(1), 173-179. doi:10.1016/j.jsurg.2016.06.001

Reeves, S., Lewin, S., Espin, S., \& Zwarenstein, M. (2010). Interprofessional teamwork for health and social care. United-Kingdom: Wiley-Blackwell.

Rokos, I. C., Sanddal, N. D., Pancioli, A. M., Wolff, C., \& Gaieski, D. F. (2010). Inter-hospital communications and transport: turning one-way funnels into two-way networks. Academic Emergency Medicine, 17(12), 1279-1285. doi:10.1111/j.1553-2712.2010.00929.x

San Martín-Rodríguez, L., Beaulieu, M.-D., D'Amour, D., \& Ferrada-Videla, M. (2005). The determinants of successful collaboration: A review of theoretical and empirical studies. Journal of Interprofessional Care, 19(sup1), 132-147. doi:10.1080/13561820500082677

Sarcevic, A., Marsic, I., Waterhouse, L. J., Stockwell, D. C., \& Burd, R. S. (2011). Leadership structures in emergency care settings: A study of two trauma centers. International Journal of Medical Informatics, 80(4), 227-238. doi:10.1016/j.ijmedinf.2011.01.004

Sethi, D., \& Subramanian, S. (2014). When place and time matter: How to conduct safe interhospital transfer of patients. Saudi Journal of Anaesthesia, 8(1), 104-113. doi:10.4103/1658-354X.125964

Sexton, J. B., Thomas, E. J., \& Helmreich, R. L. (2000). Error, stress, and teamwork in medicine $\begin{array}{lllll}\text { and aviation: cross sectional surveys. } & \text { BMJ, 320(7237), }\end{array}$ doi:10.1136/bmj.320.7237.745

Steinemann, S., Bhatt, A., Suares, G., Wei, A., Ho, N., Kurosawa, G., . . Berg, B. (2016). Trauma team discord and the role of briefing. The journal of trauma and acute care surgery, 81(1), 184-189. doi:10.1097/TA.0000000000001024

Tomizawa, R., Shigeta, M., \& Reeves, S. (2017). Framework development for the assessment of interprofessional teamwork in mental health settings. Journal of Interprofessional Care, 31(1), 43-50. doi:10.1080/13561820.2016.1233098

Twibell, R., Siela, D., Riwitis, C., Neal, A., \& Waters, N. (2018). A qualitative study of factors in nurses' and physicians' decision-making related to family presence during resuscitation. Journal of Clinical Nursing, 27(1-2), e320-e334. doi:10.1111/jocn.13948

Weller, J., Boyd, M., \& Cumin, D. (2014). Teams, tribes and patient safety: overcoming barriers to effective teamwork in healthcare. Postgraduate Medical Journal, 90(1061), 149-154. doi:10.1136/postgradmedj-2012-131168 
Westli, H. K., Johnsen, B. H., Eid, J., Rasten, I., \& Brattebø, G. (2010). Teamwork skills, shared mental models, and performance in simulated trauma teams: an independent group design. Scandinavian Journal of Trauma, Resuscitation and Emergency Medicine, 18, 47-47. doi:10.1186/1757-7241-18-47

Wood, K., Crouch, R., Rowland, E., \& Pope, C. (2015). Clinical handovers between prehospital and hospital staff: literature review. Emergency Medicine Journal, 32(7), 577-581. doi:10.1136/emermed-2013-203165

Xiao, Y., Parker, S. E., \& Manser, T. (2013). Teamwork and collaboration. In D. G. Morrow (Ed.), Reviews of human factors of ergonomics. Thousand Oaks: SAGE. 


\section{Key points}

- There are multiple factors influencing IPT that fall into five broad categories: individual, relational, processual, organizational and contextual. Individual factors, a category that is not part of the conceptual framework of Reeves et al. (2010), have emerged as being a major part of IPT.

- This study generated new results on organizational and contextual factors affecting teamwork in the trauma setting such as the organization's support and priorities, and political and economic aspects.

- These results lead to a better understanding of the realities of emergency department care of a polytrauma patient as experienced by an interprofessional trauma team and may provide tools for the future development of specific interventions to improve teamwork in this unique clinical context. 